\title{
DEVELOPMENT OF THE LESSER PERITONEAL CAVITY IN BIRDS AND MAMMALS.
}

F. MALL.

THE excellent posthumous paper of Budge, ${ }^{1}$ although incomplete, adds a great deal to our knowledge of the early formation of the pleuro-peritoneal cavity. By means of injection he filled the spaces which first appear in the chick with an aqueous solution of Prussian blue. By this method he filled the various spaces forming the cœlom as they appeared, and found that they were intimately connected with the lymphatics.

The vascular layer formed between the splanchnopleur and entoderm in birds is too well known to allow further description. According to Budge, it may be split into two layers, - a dorsal or lymphatic, and a ventral or vascular. As the first blood-vessels are formed, lymph spaces appear on their dorsal side, which flow together to form networks, and accompany the primitive veins to the axial part of the germinal area. Here the lymphatics form two spaces, one on either side of the body, which are soon connected by a bridge and thus form an $\mathrm{H}$. The cross-piece of the $\mathrm{H}$ lies oral to the sinus venosus which has just been formed. ${ }^{2}$ In its further development the sinus grows to the dorsal side of the cross-piece, thus reversing the relation of the vascular to the lymphatic system. The uprights of the $\mathrm{H}$ fall to the outside of the body, and are swallowed up in the formation of the false amnion. The cross-piece forms mainly the pericardial cavity. Shortly before the heart is formed, two diverticula grow from the cross-piece, one on either side of the chorda, towards the tail of the body, forming the primitive pleuro-peritoneal cavities. By later anastomoses, and

\footnotetext{
2 Budge, His u. Braune's Archiv, 1889 .

2 Duval, Atlas de Enbryologie, Paris, I889, gives on Pl. XVIII, 289 to 292, sectiuns through the heart region before the cross-picce of the $\mathrm{H}$ is formed. On I'l. XX, Fig. 319, and PI. XXI, Figs. 338, 339 to 340, the coelomic cavities communicate on the ventral side of the heart.
} 
finally by disappearance of the septa, the whole body-cavity is caused to communicate with the false amnion.

In this abstract I have included only what directly interests us, leaving the many interesting details regarding the development of the lymphatics.

The sprouting of the pleuro-peritoneal cavity from the pericardial places the omphalo-mesenteric veins on the ventral side of the former and on the dorsal side of the latter. After the intestinal canal is formed, it is placed on the dorsal side of the pericardial, and between the pleuro-peritoneal cavities, the septum separating the pleuro-peritoneal forming the mesentery, - a condition which remains in the adult in fishes and amphibia.

The first clear idea obtained regarding the development of the lesser peritoneal cavity and great omentum was given by Johannes Müller in I830.I To him we owe our knowledge of the mesogastrium, and the confirmation of Meckel's description $^{2}$ of the relation of the omentum to the colon.

The whole subject has been thoroughly re-worked by Toldt, ${ }^{3}$ beginning with human embryos of about the sixth week, - the same stage with which Müller started.

The present communication is intended to partly fill the gap between the works of Budge and of Toldt. This was made possible by a modification of Selenka's method by which "corrosion preparations" of very young embryos are obtained." I proceed as usual to reconstruct according to the method of Born, but instead of blending the plate, the portions which are to appear in the corrosions are cut out. If the portions cut out represent spaces, the process is simple, as for instance, blood-vessels or the cœlom. The plates are now piled, and the spaces filled with Wood's metal or plaster of Paris. The former is better, if the spaces are small. After the plaster has set, the wax may be removed by boiling in water; while, if the injection has been made with Wood's metal, the plates are simply to be pulled off. For all ordinary small models, the metal is, by all odds, the most desirable. The metal cast can now easily be polished or smoothed over with wax, and the whole buried in plaster

1 Müller, Meckel's Archiv, Bd. V.

2 Meckel, Meckel's Archiv, Bd. III.

3 Toldt, Denkschriften d. Akademie zu Wien, Bd. 4I, 1879.

- Selenka, Setzungsber. d. physiol. med. Soc. zu. Erlangen, 1886. 
of Paris, from which the metal and wax are to be removed by boiling. The mould is now to be filled with lead or solder, and the plaster broken off. By this method any portion of an embryo can be modelled in lead or solder. The method does not cost as much labor as Born's, and in many respects exceeds it in usefulness.

\section{Birds.}

Shortly after the heart is formed, the head begins to flex upon the body, and the pharyngeal entoderm is pushed at first to the dorsal side of the heart, and then into the branchial region. At the time this peculiar rotation is taking place, the sinus venosus holds quite a stationary position, while the heart is thrown over to its ventral and aboral side. At this time a single omphalo-mesenteric vein empties into the heart, and from either side an additional branch, the ductus Cuvieri, is added. The pleuro-peritoneal cavity now breaks through on the aboral side of the ductus, thus causing the vessel to perforate the pleuro-peritoneal cavity (Fig. I), as described by Balfour. ${ }^{1}$ The union of the cavities across the median line now extends more aboralwards, and then communicates with the false amnion. After we have arrived at this stage, the mesentery is fully formed, the cavities on either side communicating across the median line only in the neighborhood of the heart. The pulmonary buds are forming, and the alimentary canal is almost completely closed. Just at this time two sacs, one on either side, are formed from the splanchnic wall of the pleuro-

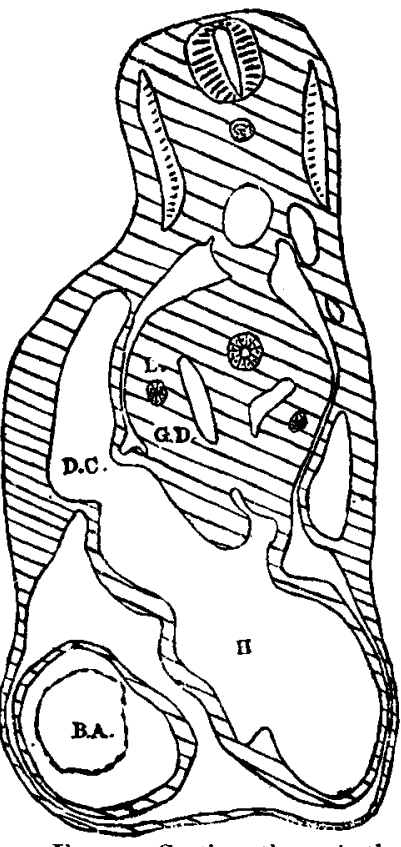

FIG. I. Section through the region of the heart of a chick of 70 hours. Enlarged 5o times. The section strikes the oral end of the gastric diverticulum, G.D., and the tips of the pulmonary buds, $L$. A few sections deeper the gastric diverticula communicate with the pleuro-peritoneal cavity. $H$, heart; $D . C$., ductus Cuvieri; $B$. A., bulbus aortæ.

1 Comparative Embryology, Vol. II, p. 627, Fig. 352. See also His, Hünschen Monograph., Taf. XI, Figs. I, 12, 13, 14; Duval, Planche. XXII, Figs. 358, 359, 360: PI. XXV, Figs. 403-407. 
peritoneal cavity. They extend between the proventriculus and the lungs, as shown in Fig. I, G. D. On account of their location, I shall speak of these sacs as the gastric diverticula. ${ }^{1}$ From the one on the right side the lesser peritoneal cavity of birds and mammals develops.

A few hours later, eighty-eight hours, when the body is flexed to its maximum, the gastric diverticula become more semicircular in transverse section, and also enclose the proventriculus on either side. On the right side the diverticulum is larger, markedly cup-shaped, and connects by means of a narrowed opening with the right pleuro-peritoneal cavity (Fig. 2, F. W. .). ${ }^{2}$

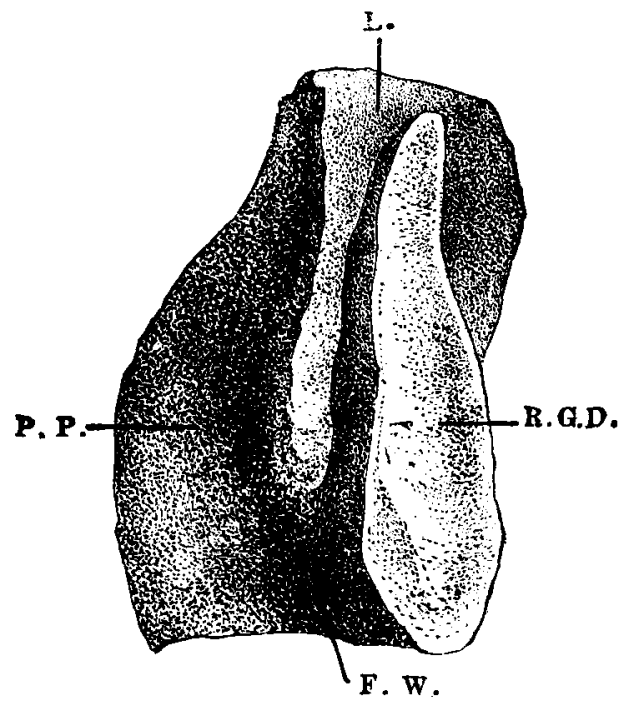

FIG. 2. Corrosion preparation of the right gastric diverticulum of a chick, 88 hours. Enlarged 44 diameters. P.P., pleuro-peritoneal cavity; $R . G . D$., right gastric diverticulum; $F$. W., foramen of Winslow; $L$., position of the right lung.

In this embryo the liver lies between the heart and proventriculus, the opening lying to the oral and dorsal side of the liver. The cœliac axis is already formed, and the gastric and hepatic arteries pass directly to the proventriculus and liver. On account

1 Earlier stages are shown in Duval, Pl. XXV, Figs. 404 and 405 . His sections are viewed from the aboral side. The communication with the pleuro-peritoneal cavity of the stage which I represent in Fig. I, he shows on Pl. XXX, Figs. 473 to 476.

2 Duval, Pl. XXX, Figs. 375, 376, shows a section of the communication of this opening with the coelom. 
of this relation, and on account of a similar arrangement in mammals, I conclude that this constriction marks the foramen of Winslow; and I shall speak of it as such.

On the left side (Fig. 3) the gastric diverticulum is much

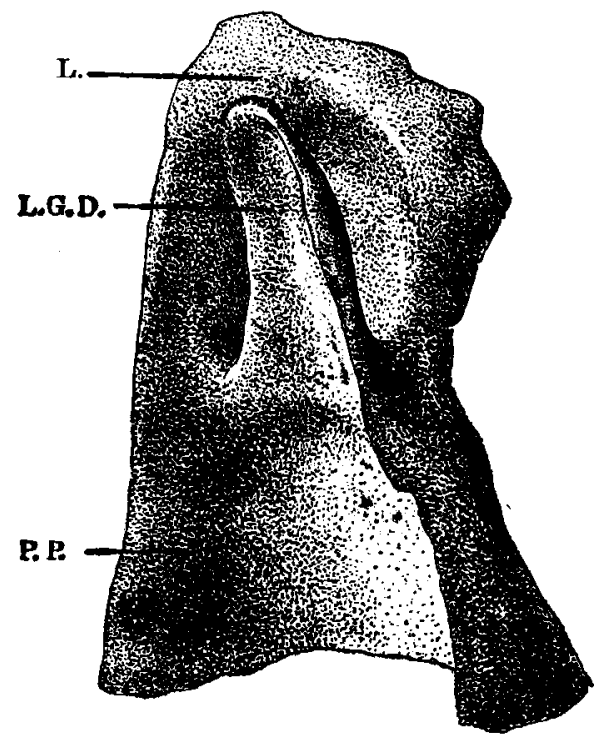

Fig. 3. Left gastric diverticulum of a chick, 88 hours. Enlarged 44 diameters. P.P., pleuro-peritoneal cavity; L.G.D., left gastric diverticulum; $L$., position of left lung.

shorter and more shallow. In general, the proventriculus lies on the left side of the median line, and the spaces on either side seem to have taken on a corresponding shape and capacity.

In an embryo of five days and sixteen hours the right gastric diverticulum has about doubled all its diameters, while the embryonic foramen of Winslow has become much more sharply defined $\left(\mathrm{Fi}_{2}\right.$. 4). At this time the liver has greatly increased in size, the right lobe being larger than the left, both lying oral to the foramen of Winslow. The original position of the liver being aboral to the foramen, the rotation of the liver necessarily carries the hepatic artery and the portal vein around the foramen of Winslow, its adult position. With the growth of the gizzard, a space extends from the aboral end of the right gastric diverticulum along the dorsal side of this organ, and marks the beginning of the cavity of the great omentum (o). Nearly the whole 
[VoL. V.

of the right diverticulum now lies on the left side of the body; the same position is held by the proventriculus and gizzard. ${ }^{1}$

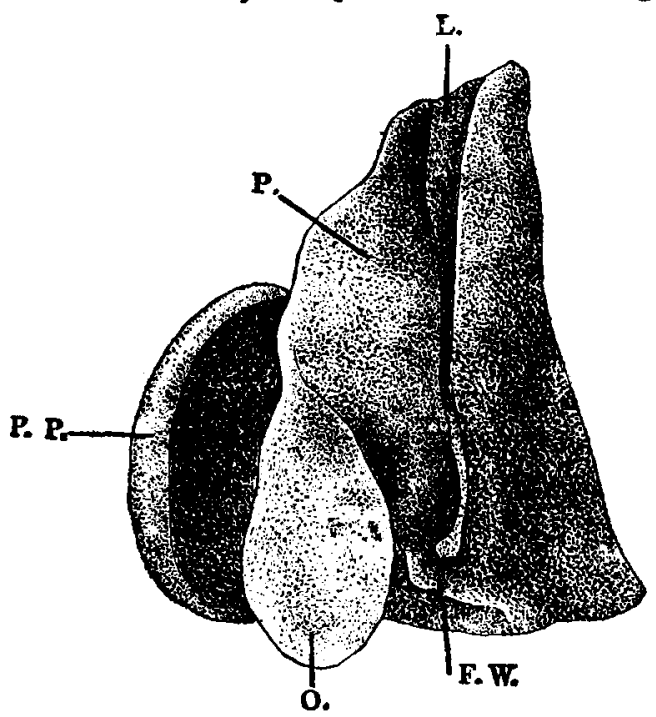

FIG. 4. Corrosion preparation of the right gastric diverticulum in a chick of 136 hours. Ventral view. Enlarged 22 times. $P . P$., pleuro-peritoneal cavity; $F . W$., foramen of Winslow; $L$, position of right lung; $P$., position of proventriculus; $O$., omental cavity : position of gizzard.

On the left side the gastric diverticulum has become but slightly larger than in the previous stage (Fig. 5). It commu-

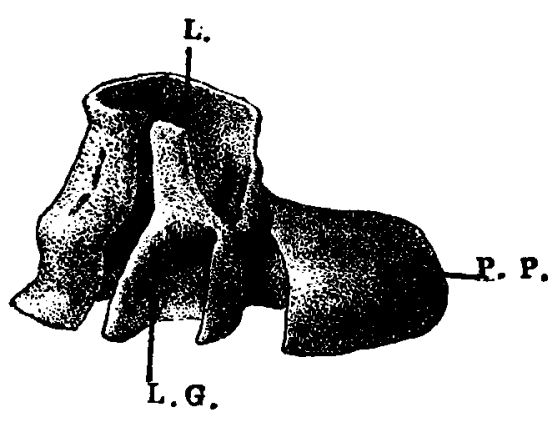

Fig. 5. Corrosion preparation of the left diverticulum in a chick of 136 hours. Ventral view. Enlarged 22 times. $P$. $P$., pleuroperitoneal cavity; $L$. $G$., left gastric diverticulum; $L$, position of left lung. nicates on its aboral side most freely with the pleuroperitoneal cavity, and no indication of a constriction (foramen of Winslow) is at any time present.

A day later (six days, sixteen hours) the left diverticulum has nearly disappeared, while the dimensions of the right have increased considerably in size. In general, its form is much as shown in Fig. 4. The lobus

1 Duval, P1. XXIV, Figs. 527 to 535 , represents sections of this stage. The sections are all viewed from the aboral side; that is, they are negative pictures. 
Spigelii now projects markedly into the oral portion of the diverticulum. The portion on the dorsal side of the gizzard has increased with the growth of this organ, and also extends markedly into the omentum which partly overhangs the intestine. The whole diverticulum measures, from lung to intestine, three millimetres; and the gizzard is one millimetre in diameter. The omental cavity is nearly a millimetre wide, and about one-half a millimetre long. It is shown as a distinct cavity, the dorsoventricular diameter being nearly half a millimetre.

My material does not suffice to follow the fate of the embryonic omentum. A chick of ten days shows, however, that it is in no way related to the formation of the air sacs. At this stage three large air sacs project from the bronchial tubules into the abdominal cavity, representing the number present in the adult.

The domestic fowl contains three peritoneal cavities, completely separated from one another, and from one of them the lesser cavity arises. The communication is by means of a foramen of Winslow. After the anterior abdominal walls are removed, two ventral cavities are exposed, separated from each other by a vertical longitudinal septum. Into each of the cavities hangs a lobe of the liver, while into the left the ventral surface of the gizzard projects. On the dorsal side of the liver there are, on each side, three air sacs, - an anterior, a middle, and a posterior. The middle is the smallest, and the posterior the largest, and extends throughout the posterior portion of the abdominal cavity. Each sac communicates by means of a special opening directly with the lungs. By allowing the sacs to collapse, we find that a very distinct membrane extends backward from the gizzard, and cuts off the portion of the abdominal cavity containing the intestines. This is the "pseudo-epiploön" described by Weldon, ${ }^{1}$ and followed more extensively by Beddard. ${ }^{2}$ A similar membrane is present in crocodiles. In all respects, this membrane is situated in the same position as the epiploön in mammals, with the difference that it is adherent to the abdominal walls along its free border. On the dorsal side of the gizzard the air sacs fill all the space, thus closing off the communication between the cavity in which the intestines lie and the ventral cavities.

1 Proceedings of the Zoölogical Society, 1883.

2 Jiid., 1885 . 
When the anterior and middle air sacs on the right side are separated from the posterior sac on the same side (which is quite easily done with the handle of the scalpel), a slit is shown which extends to the oral and dorsal sides of the hepatic veins. Here it communicates, by means of a round opening about one centimetre in diameter, with a large cavity lying on the median and dorsal side of the proventriculus and extending to the spleen. The cavity does not extend over the dorsal side of the gizzard. In all respects, this corresponds with the right gastric diverticulum and with the lesser peritoneal cavity in mammals.

The relation of this embryonic omentum to the "pseudoepiploön" is as yet unknown. However, I think it probable that the one is changed into the other, and that the "pseudoepiploön will prove to be the true epiploön, homologous with the same in mammals. We must only imagine the embryonic omentum attaching itself to the sides of the abdomen, followed by a loss of the epiploönic peritoneal cavity. A subsequent growth of the air cells back from the dorsal side of the stomach will produce the condition found in the adult.

\section{MammaLs.}

The earliest stage of mammals I have to examine is an embryo of a dog, six millimetres long. ${ }^{1}$ It represents about the same stages as the chick of seventy hours already described. In the chick the pericardial cavity communicated on all sides with the pleuro-peritoneal cavity, while in this specimen the communication takes place only on the aboral side of the sinus reunieus. His pictures sections of human embryos, both earlier and later than this stage $;^{2}$ and in all of them the communication of the pericardial cavity with the pleuro-peritoneal cavity is only on the oral side of the ductus Cuvieri. The cœlom still communicates freely with the false amnion.

The stomach is still a straight, upright tube, communicating freely behind with the umbilical vesicle. The liver is just arising as two lateral spouts of epithelial cells, each of which encircles an omphalo-mesenteric vein.

\footnotetext{
1 For description of this embryo, see my paper on "Branchial Clefts of the Dog," Studies from the Biological Laboratory, Fohns Hopkins University, Vol. IV.

2 Anatomic menschlich. Embryonen, Atlas. Earlier stages, Pl. XI, $B B$ and $L R$; later stages, PI. XII, $R$; II, $B$; IV, $A$.
} 
On the right side a marked gastric diverticulum is present, as shown in Fig. 6. On the left side absolutely no diverticulum is present. The same holds true for all the sections pictured by Professor His in his Atlas. Al. though there are two gastric diverticula in the chick, I have found in a specimen of sixty hours a single right diverticulum, much like the one shown in Fig. 6. This may indicate that the left diverticulum is in process of degeneration.

In a human embryo seven millimetres long the gastric diverticulum is slightly larger than the dog just described. It corre-

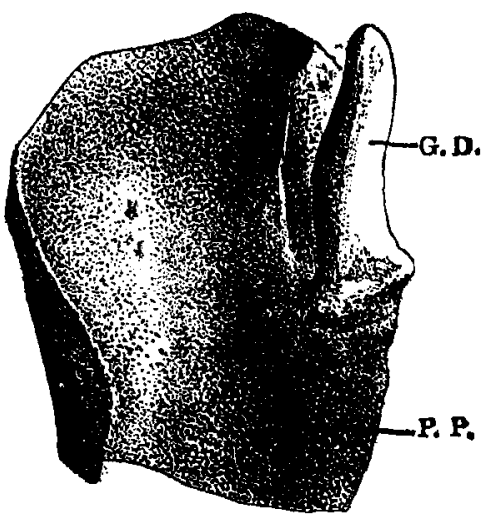

Fig. 6. Corrosion preparation of a portion of the right cœlom, including the gastric diverticulum of the $\operatorname{dog} 6$ millimetres long. Ventral view. Ensponds about with His's $A, B$, larged 66 times. G. $D$., gastric diverand $B r_{3 .}{ }^{1}$ The embryo was cut ticulum; $P . P$., pleuro-peritoneal cavity. into a perfect series, and $I$ have reconstructed in wax the whole embryo. A cast of the cœlom was made with Wood's metal.

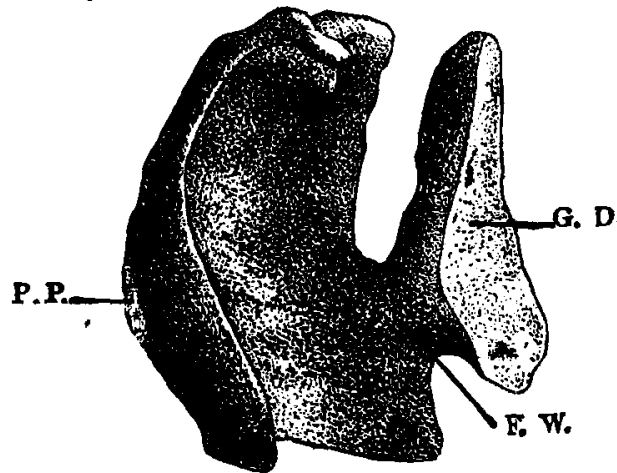

FIG. 7. Corrusion preparation of the gastric diverticulum of a human embryo 7 millimetres long. Enlarged 44 times. P.P., pleuro-peritoneal cavity; $F$. W., foramen of Winslow; G. D., gastric diverticulum. phalo-mesenteric vessels into the cord. On the ventral side of the alimentary canal, both on the oral and aboral sides of the

\footnotetext{
1 Anatomie menschlich. Embryonen, and Ablandl. d. K. S. Ges. d. Wiss., Bd. XIV, 1888.
} surrounds the heart with the exception of the point where the sinus reuniens enters. The bulbus aorta is completely surrounded by a space to its origin in the ventricle. The cavity communicates on either side with the pleuro-peritoneal. It then surrounds the intestine, liver, and extends about the om- 
omphalo-mesenteric vessels, the cavities on either side communicate by means of two quite large openings. ${ }^{1}$

Fig. 7 shows the gastric diverticulum somewhat more advanced than in Fig. 6, and resembling greatly Fig. 2. The marked constriction of the connection of the diverticulum with the pleuro-peritoneal cavity already marks the foramen of Winslow. A transverse section through the foramen of Winslow is shown in Fig. 8. The section strikes the cœliac axis as it arises

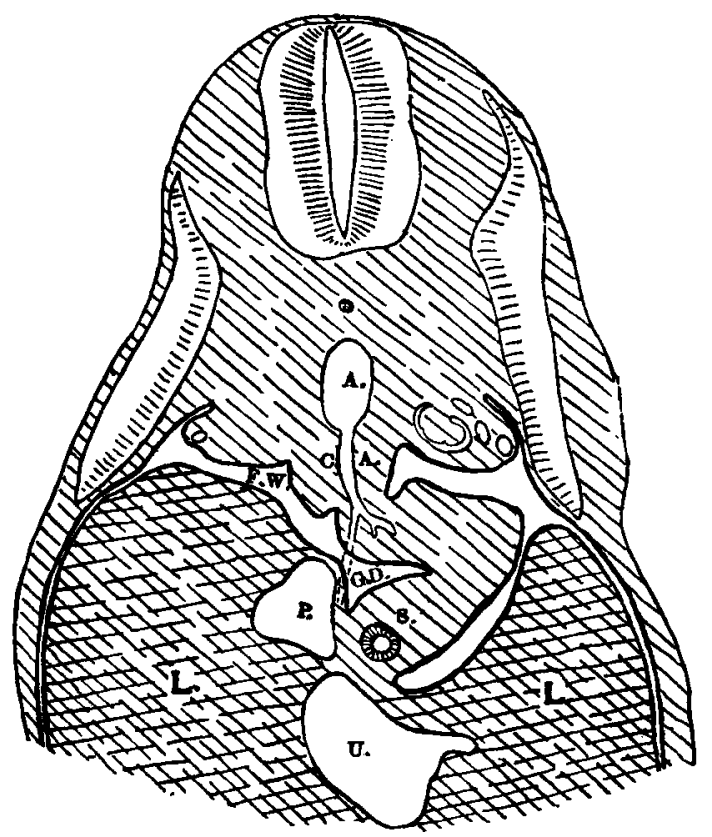

Fig. 8. Section through the foramen of Winslow of a human embryo 7 millimetres long. Enlarged 50 times. $A$., aorta; $C . A$., coeliac axis; $S$., stomach; $G . D$., gastric diverticulum; $F . W$., foramen of Winslow; $P$., portal vein; $U$, umbilical vein; $L$., liver.

from the aorta. The dotted line indicates the course of the hepatic artery a few sections deeper. It passes to the aboral side of the foramen, and on the median side of the vein the relations which are retained in the adult.

The mesentery has become considerably larger than in the dog of six millimetres or in the chick of seventy hours (Fig. I). Accompanying the increased length of the mesentery, there is

1 A description of this embryo will be given in the next number of this Journal. 
a corresponding increase of the lesser peritoneal cavity, which now lies on the dorsal side of the embryonic stomach. Besides the elongation of the mesentery, there is also a gradual shifting of the stomach away from the mouth. After the liver and heart are well formed they lie in a straight line, in a transverse section, the liver being between the stomach and the heart. These three organs now lie immediately on the ventral side of the first dorsal vertebra. In the adult the stomach lies below the twelfth dorsal. In the dog six millimetres long there is as yet no artery to these organs, while in the human, seven millimetres, the artery is well formed, and arises from the aorta, just opposite the fourth dorsal. The omphalo-mesenteric artery, i.e. superior mesenteric, arises one segment deeper. In two dogs ten millimetres long the cœliac axis arises opposite the second and fourth dorsal segments respectively, while in a dog, 13.5 millimetres long, it lies as low as the tenth. In a cat twelve millimetres the coliac axis lies opposite the tenth dorsal, and the omphalo-mesenteric artery opposite the eleventh dorsal. In His's embryo $M$ the omphalo-mesenteric arises opposite the first dorsal, ${ }^{1}$ while in embryo $B$ the same vessel arises opposite the fifth dorsal. ${ }^{2}$ In embryo $A$ the coliac axis arises opposite the sixth dorsal, and the omphalo-mesenteric opposite the ninth dorsal. ${ }^{3}$

These cases are sufficient to show that the stomach gradually moves away from the mouth, carrying its artery with it. Additional evidence is obtained from the study of the development of the splanchnic nerve. When the cœliac axis is opposite the first and second dorsal vertebræ, there are twigs of sympathetic nerves which surround it, coming from the first and second dorsal nerves. As the artery descends, the nerves following, third, fourth, etc., are, one after another, included, thus accounting for the high origin of the splanchnic.4

In a dog ten millimetres long the general shape of the lesser peritoneal cavity corresponds with the outline of the stomach.

1 Atlas, PI. VII, M 4; PI. VI, Fig. I5.

${ }^{2}$ Replace Fig. 24, Pl. III, Atlas, into Fig. I, PI. I.

${ }^{8}$ Replace Figs. 79 and 86, Pl. V, Allas, into Fig. 4, Pl. I.

Although the splanchnic is usually spoken of as arising from the eighth dorsal, it may be traced up to the third, second, or even the first dorsal. - Beck, Phil. Trans., 1846.

The change of the relation of the spinal column to the heart is shown in a figure by Uskow, Arch. für mikrosk. Anat., Bd. 22, S. 188. 
The communication with the pleuro-peritoneal cavity has become constricted to a narrow canal, as shown in Fig. 9. The cavity

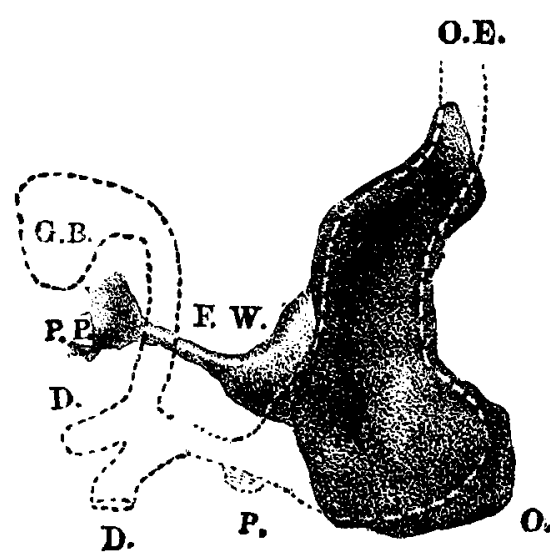

Fic. 9. Corrosion preparation of the gastric diverticulum (lesser peritoneal cavity) of a dog 10 millimetres long. Enlarged 44 diameters. The view is from the left side, and the stomach is outlined. $E$., cesophagus; $G$. B., gall bladder; $P$., pancreas; $D$., duodenum; $D$., second canal to the liver; $P$. $P$., pleuro-peritoneal cavity; $F$. $W$., foramen of Winslow; $O$, omental cavity.

itself is still pointed on its oral side, extending up to the lung. On the aboral side it extends beyond the greater curvature of the stomach, forming the omental cavity. The liver at this stage is composed of two lobes, the right slightly larger than the left, and connected with each other by a complete band of liver tissue. A lobus Spigelii, as exists in birds, has not yet formed.

A stage later (Fig. IO), the stomach has twisted about, holding in great part its adult position. The liver, which before lay on the ventral side of the stomach, now lies on the aboral, and in great part on the dorsal side. The gall bladder, which before lay in the septum transversum on the ventral side of the stomach

$$
\text { B.D. }
$$

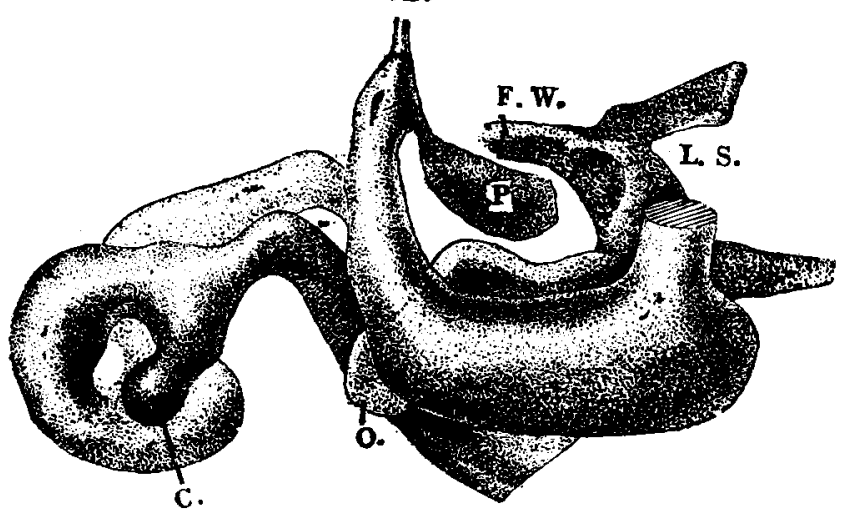

FIG. Io. Stomach, intestines, and lesser peritoneal cavity of a dog 13.5 millimetres long. Viewed from the left side. Enlarged 22 times. The lesser peritoneal cavity has been filled with metal. B.D., bile duct; $P$., pancreas; $C$., çæcum; $O$., omentum; F. W., foramen of Winslow; L. S., position of the lobus Spigelii. 
and the aboral side of the liver, now lies on the aboral side of the stomach and on the ventral side of the liver. It has also shifted its position to the right of the median line. A model of these parts, from the ten-millimetre embryo, simply rotated, to throw the stomach away from the mouth, gives the condition of things as they exist in the embryo thirteen millimetres long. The intestine is composed of two loops, one from the stomach, extending into the pelvis, and the other at the crcum, extending to the stomach, and on the left side of the first loop. The second loop is shown in Fig. Io.

The rapid growth of the large intestine has thrown the cacum as high as the stomach, but to the right of it. The fold of

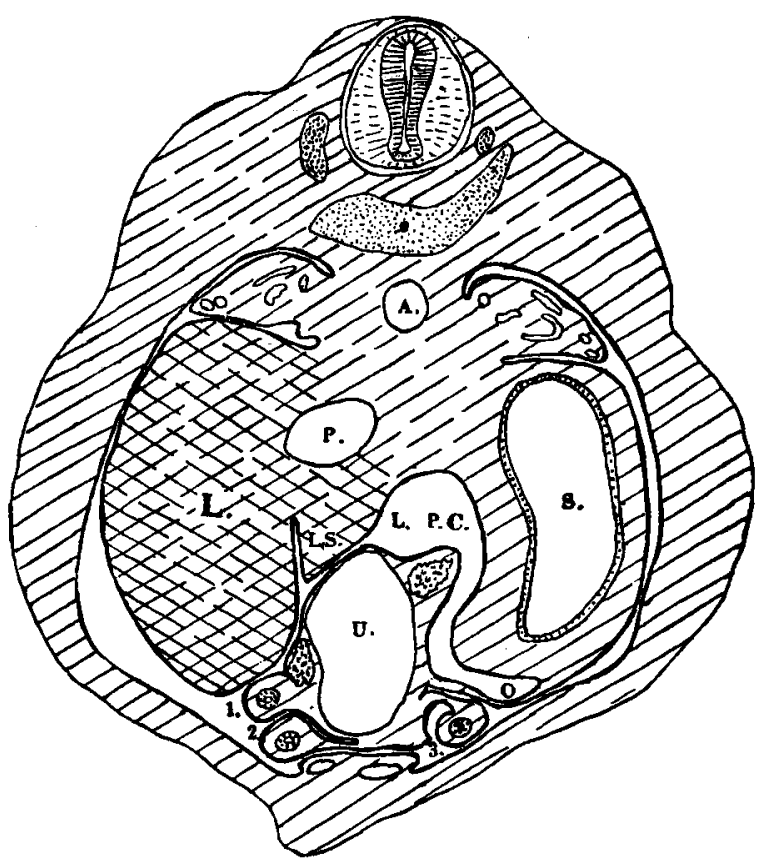

FIG. II. Section through the foramen of Winslow and the lobus Spigelii of a dog 13.5 millimetres long. Enlarged 36 times. $A$., aorta; $S$., stomach; $P$, portal vein; $U$., umbilical vein; 1,2 , and 3 , descending, ascending, and descending portions of the intestine; L.P.C., lesser peritoneal cavity; $L . S$., lobus Spigelii; $O$, omental cavity.

omentum, coming from the dorsal side of the stomach, passes over the large intestine, as shown in the figure. The general shape of the lesser peritoneal cavity is shown in Fig. II. The 
S-shaped loop of intestine is cut across three times, and the lesser peritoneal cavity with its communication is shown throughout its whole extent. Within the foramen of Winslow, or rather the communication of the lesser with the greater peritoneal cavities, lies a triangular lobe of the liver, the lobus Spigelii. It arises from the right lobe of the liver, and is surrounded with a space, as shown in Fig. IO. In many respects, the liver of this stage represents the condition found in the adult domestic fowl.

The omentum from now on rapidly grows, covering all the intestines, but not adhering to the colon, as is the case in man. For the later development I refer the reader to the excellent papers of Müller and of Toldt.

In the lower vertebrates the alimentary canal is attached dorsally by quite a simple mesentery. The same is the case in the embryos of all vertebrates. From this simple, straight mesentery, by means of shifting, the later compartments of the cœlom are formed. In the lizard the mesentery is quite simple, ${ }^{1}$ and lately Ravn has shown us that on either side of the stomach there is a sack which communicates with the peritoneal cavity, the blind end extending as far forward as the heart. ${ }^{2}$ On the right side the opening of the sac is related to the surrounding viscera, as is the foramen of Winslow in birds and mammals. The development of these sacs is not known, but from sections of embryos given by Orr a marked gastric diverticulum is shown. ${ }^{3}$ In some specimens of adult turtles which $I$ have examined there is a peritoneal cavity at the lesser curvature of the stomach, which does not communicate with the greater peritoneal cavity. This cavity holds the position of the lesser cavity, and no doubt will prove to be such. Fishes and amphibia probably have no such cavity.

From these observations it is seen that the lesser peritoneal cavity is present in reptiles, birds, and mammals. In certain groups of reptiles two diverticula are formed, extending from the pleuro-peritoneal cavity, on either side of the stomach, to the heart. The right is larger than the left, and communicates with the colom around the vessels passing to the liver. This

1 Pittard, Todd's Cyclopadia of Anatomy, Vol. III.

2 Ravn, His u. Braune's Archiv, 1889.

8 Orr, Fournal of Morphology, Vol. I, PI. XIV, Fig 37, c, PC. 
cavity is homologous with the lesser peritoneal cavity, and the opening with the foramen of Winslow of mammals. Both right and left cavities are present in the adult.

In birds it plays a most important part, forming a distinct cavity, homologous with the lesser peritoneal cavity in mammals. At first the cavities are symmetrical, one on either side, but later in the development the left. one disappears, and the right gradually enlarges to form the lesser peritoneal cavity, while the communication about the hepatic vessels with the greater cavity forms a foramen of Winslow.

In the lower mammals there are two gastric diverticula (Ravn), but the left is very insignificant and soon disappears. In the higher mammals only the right one is formed, which gradually increases in size to form the lesser peritoneal cavity.

Clark University, April 6, i8gi. 